\title{
MicroRNAs in Oligodendrocyte and Schwann Cell Differentiation
}

\author{
Jason C. Dugas ${ }^{a}$ Lucia Notterpek ${ }^{b}$ \\ a Department of Neurobiology, Stanford University, Stanford, Calif., and b Department of Neuroscience, \\ College of Medicine, McKnight Brain Institute, University of Florida, Gainesville, Fla., USA
}

\author{
Key Words \\ miRNA $\cdot$ Oligodendrocyte $\cdot$ Schwann cell $\cdot$ Myelin
}

\begin{abstract}
MicroRNAs (miRNAs) are a class of small (approx. $22 \mathrm{nt}$ ) noncoding RNAs that are capable of post-transcriptionally silencing mRNAs that contain sequences complementary to the miRNAs' 7- to 8-bp 'seed' sequence. As single miRNAs are often predicted to target up to hundreds of individual transcripts, miRNAs are able to broadly affect the overall protein expression state of the cell. This can translate into global effects on cellular health and differentiation state. Recently, several reports have identified crucial roles for miRNAs in controlling the production, differentiation, and health of myelinating cells of the mammalian nervous system. In this review, we will discuss how individual miRNAs regulate these various processes, and also how miRNA production in general is required for several stages of myelin generation and maintenance.

Copyright $\odot 2011$ S. Karger AG, Basel
\end{abstract}

\section{Oligodendrocytes Require MicroRNAs at Various Stages of Development}

MicroRNAs (miRNAs) are a class of small (approx. 22 nt) noncoding RNAs that are capable of post-transcriptionally silencing mRNAs that contain sequences complementary to the miRNAs' 7 - to 8-bp 'seed' sequence
[Bartel, 2004; Wu and Belasco, 2008]. To study the overall role of miRNAs in biological processes, researchers have knocked out enzymes required for normal miRNA processing, such as Dicer1 [Bartel, 2004]. However, Dicer1 ${ }^{-/}$ mice fail to survive past embryonic day 7.5 (E7.5), preventing temporal developmental studies [Bernstein et al., 2003]. Therefore, to study the requirement of mature miRNAs in postnatal processes, Cre-mediated recombination has been used to disrupt Dicerl function specifically in cells of interest. In this way, functional miRNAs have been shown to be required at all stages of oligodendrocyte $(\mathrm{OL})$ generation and myelination in the mammalian central nervous system (CNS). Knockout of Dicerl function in uncommitted neural precursors, by driving Cre expression from the Nestin promoter, leads to a reduction in overall OL lineage cell number, both mature OLs and immature OL precursor cells (OPCs) [KawaseKoga et al., 2009]. This may represent a reduction in OPC generation from neural precursors, as opposed to OPC proliferation, because disruption of Dicerl function in specified OPCs and OLs by expressing Cre from either the Olig1 or Olig2 promoter does not reduce OPC number in vivo [Dugas et al., 2010; Zhao et al., 2010]. However, OPCs that lack Dicerl do fail to differentiate normally, as OL differentiation and myelin formation are significantly disrupted in Olig1-Cre, Olig2-Cre, and CNP-Cre; Dicer$1^{\text {Flox/Flox }}$ mice, and OPCs purified from these animals fail to differentiate normally in vitro. Finally, mature miRNA production is not only necessary during development, but is also required to maintain healthy myelin, as dis-

\section{KARGER}

Fax +4161306 1234

E-Mail karger@karger.ch

www.karger.com (c) 2011 S. Karger AG, Basel

Accessible online at: www.karger.com/dne
Jason Dugas

Department of Neurobiology, Stanford University

D205 Fairchild Building

299 Campus Dr. West, Stanford, CA 94305-5125 (USA)

Tel. +1 650736 8561, E-Mail jcdugas@ alum.mit.edu 
Fig. 1. miRNA regulation of CNS myelination. Specific miRNAs involved in regulating various stages of OL differentiation and myelination are shown (or miRNAs in general in the neural precursor to OPC transition). Targets of miRNAs are shown, with confirmed targets shown in bold and predicted targets in plain text. miR-19b is expressed in both OPCs and OLs, miR-9 is more highly expressed in OPCs, and all other miRNAs shown are more highly expressed in OLs. Inhibition of expression/ stage transition is shown by lines with bars, activation of expression/stage transition is shown by lines with arrows, and expression that may be detrimental to cell health/ function is shown by lines with circles.

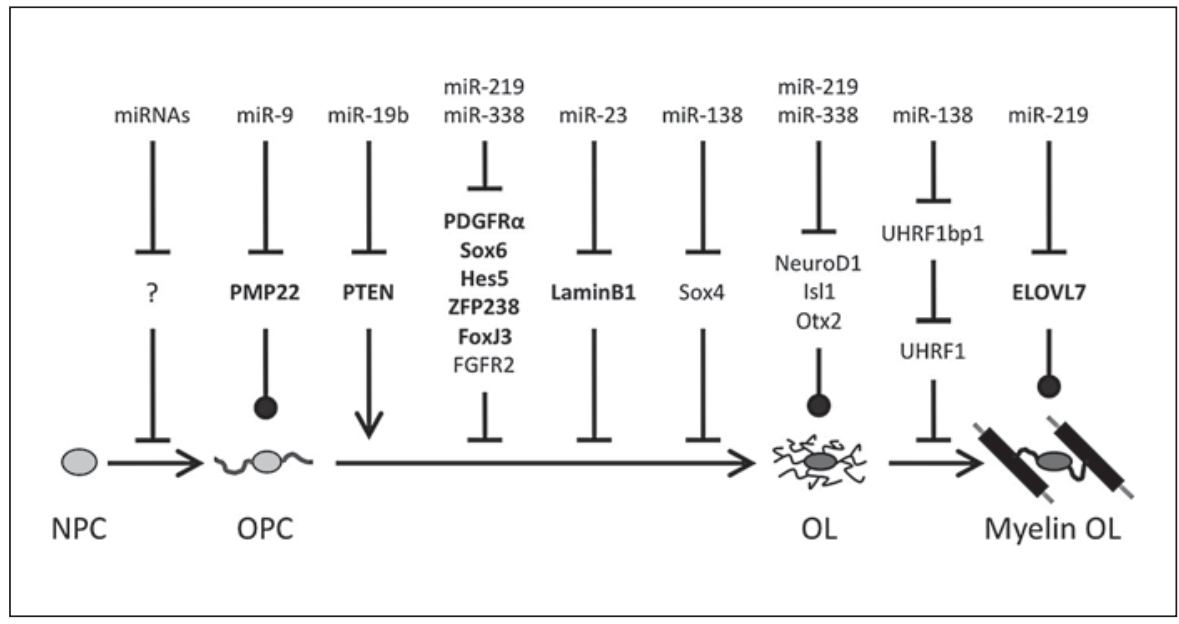

ruption of Dicerl specifically in fully mature OLs, by driving tamoxifen-inducible Cre expression from a PLP promoter, leads to the eventual degradation of fully formed CNS myelin [Shin et al., 2009]. In summary, these results indicate that mature miRNA activity is required at various stages of OL development, including in the initial production of fate-specified OPCs, in the differentiation of mature OLs and generation of compact CNS myelin during development, and in the maintenance of functional myelin sheaths in adult animals (fig. 1).

\section{The Influences of Individual miRNAs on OL Biology}

Having determined that mature miRNAs are required for normal OL generation and myelin formation, several groups have subsequently investigated the roles of individual miRNAs in promoting functional CNS myelination. These experiments have identified specific miRNAs that promote the formation and maintenance of healthy CNS myelin by four distinct mechanisms: the suppression of OPC-expressed genes to promote differentiation, the promotion of OPC expansion, the overall suppression of inappropriate non-OL lineage gene expression in OPCs and OLs, and the suppression of genes transiently required at high levels during myelin sheath formation.

\section{miRNA Promotion of OL Differentiation}

The work of Dugas et al. [2010] and Zhao et al. [2010] indicates that miRNAs are critically required for the normal process of OL differentiation. To begin to identify the specific miRNAs that may be promoting OL differentiation, both labs independently identified miR-219 and miR-338 as two of the most highly induced miRNAs in differentiating OLs, a finding consistent with previous characterizations of miRNA expression in OLs [Lau et al., 2008]. In addition, miR-219 expression appears to be restricted to the vertebrate CNS, and to mature OLs within the brain and spinal cord [Dugas et al., 2010; Wienholds et al., 2005], indicating that miR-219 is highly enriched within OLs relative to all other vertebrate tissues. Interestingly, miR-338 expression appears to be less widespread in the CNS, as strong expression of miR-338 is only detected in the spinal cord, with significantly weaker signal in the brain [Dugas et al., 2010; Zhao et al., 2010]. Functionally, both miR-219 and miR-338 are individually necessary and sufficient to promote normal OPC differentiation into OLs in vitro and in vivo. miR-219 appears to exert these effects, at least in part, by suppressing the production of several OPC-expressed proteins that normally hinder OL differentiation: PDGFR $\alpha$, the receptor for the OPC mitogen PDGF, is directly suppressed by miR-219, as are the differentiation-inhibiting transcription factors Sox6, Hes5, ZFP238, and FoxJ3 [Dugas et al., 2010; Zhao et al., 2010]. Sox6, Hes5, and ZFP238 are similarly repressed by miR-338, and in addition a second OPC mitogen receptor, FGFR2, is also a predicted target of miR-338. It is worth noting that while Zhao et al. [2010] were able to detect a functional role for miR-338 in promoting OL differentiation, this finding was not reproduced by Dugas et al. [2010]. This discrepancy may in part result from the fact that Dugas et al. only used miR338-5p in their experiments, whereas Zhao et al. used both miR-338-5p and $-3 p$. It is the $3 p$ strand that targets 
FGFR2 and ZFP238, and therefore the 3p strand of miR338 may significantly contribute to the promotion of OL differentiation observed in the latter study.

In addition to miR-219 and miR-338, miR-23a and miR-23b have been identified as miRNAs that are both induced approximately 5-fold during OL maturation, and overexpression of either can enhance OL differentiation [Lau et al., 2008; Lin and Fu, 2009]. miR-23 represses Lamin B1 (Lmnb1) expression, a gene which is normally downregulated during OL differentiation, and overexpression of which inhibits the normal morphological differentiation of OLs [Lin and Fu, 2009]. Furthermore, Lmnb1 duplication in humans leads to late loss of healthy myelin in autosomal dominant leukodystrophy [Padiath et al., 2006]. Therefore, miR-23 appears to also influence OL differentiation by reducing the expression of a gene that inhibits normal OL maturation.

Cumulatively, these data indicate a model in which a prominent role of OL-induced miRNA expression is to link the initiation of OL differentiation to the suppression of OPC proliferation. Upon the initiation of differentiation, not only must several genes specific to mature OLs be induced, but OPC-expressed genes involved in promoting proliferation and blocking differentiation must simultaneously be suppressed. By strongly inducing miR219 , miR-338, and miR-23 at the outset of OL differentiation, the cell is able to rapidly suppress the production of several genes that normally maintain the OPC in a proliferative, undifferentiated state, thereby facilitating the rapid transition from proliferating precursor to postmitotic, differentiated OL. Indeed, the model of miRNA function as increasing the gain of a developmental state change has been postulated previously [Bartel, 2009; Reinhart et al., 2000], and therefore these results likely represent one general mode of action of miRNAs in development.

Interestingly, miR-138, which is also induced in differentiating OLs, appears to play a distinct role in regulating OL differentiation. OL differentiation proceeds in a series of discrete temporal stages [Baumann and PhamDinh, 2001; Dugas et al., 2006]. Whereas miR-219 promotes all stages of OL differentiation, miR-138 specifically promotes the early stages (indicated by expression of myelin proteins CNP and MBP) of OL differentiation, while simultaneously suppressing the later [myelin oligodendrocyte glycoprotein (MOG) expressing] stage of OL differentiation [Dugas et al., 2010]. This intermediate $\mathrm{MBP}^{+} / \mathrm{MOG}^{-}$stage corresponds to the point at which early differentiating OLs extend processes to contact axons and initiate myelin sheath formation; by the time OLs are $\mathrm{MOG}^{+}$, they have lost the ability to form new myelin sheaths [Watkins et al., 2008]. Potentially, miR-138 could play a central role in lengthening this intermediate stage of OL differentiation, which would extend the time frame in which a newly differentiating OL could form the contacts that will produce mature myelin sheaths. How miR138 accomplishes this role remains unclear, but potentially miR-138 could simultaneously target one set of genes that represses the initiation of OL differentiation and another set of genes that promotes the late stage of differentiation. Candidates include Sox4, which pairs with Sox6 to inhibit early OL differentiation [Stolt et al., 2006], and UHRF1bp1, a putative binding partner of UHRF1, which itself has been shown to specifically inhibit the late phase of OL differentiation [Dugas et al., 2006].

\section{miRNA Promotion of OPC Expansion}

In contrast to the identified role of several miRNAs in promoting OL differentiation, at least one group has identified a set of OPC-enriched miRNAs that appear to impede OL differentiation. Budde et al. [2010] found that several members of the miR-17-92 cluster were expressed at high levels in both OPCs and OLs, and that members of this cluster were both necessary and sufficient to enhance OPC proliferation in vivo and in vitro. Furthermore, they found that a single member of this cluster, miR-19b, could increase OPC proliferation in vitro, and that this correlated with the suppression of the predicted miR-19b target PTEN and an associated downstream increase in Akt phosphorylation, which could account for the activity of miR-19b [Paintlia et al., 2010]. These data clearly illustrate that individual miRNAs can play contradictory roles in regulating differentiation (miR-19b and related miRNAs promote OPC proliferation, whereas miR-219, miR-338, and miR-23 promote OL differentiation), and demonstrate that the dynamic regulation of individual miRNAs is likely as important in the control of differentiation as the regulation of individual proteins.

\section{miRNA Suppression of Inappropriate Gene Expression}

Often, the expression patterns of genes targeted by an miRNA are inversely correlated with the expression of the targeting miRNA, e.g. as miR-219 levels rise in differentiating OLs, the expression of several genes targeted by 
miR-219 falls [Dugas et al., 2010]. However, this is not always the case. In one of the earliest studies of miRNA expression in OL lineage cells, miR-9 was one of two OPCenriched miRNAs identified whose expression was positively correlated with its predicted targets [Lau et al., 2008]. This interrelated expression pattern may indicate a role in repressing inappropriate gene expression; namely, miR-9 may be expressed to silence the 'leaky' expression of genes that should not be produced in OL lineage cells, and would therefore be required at highest levels when its targeted genes are also being most highly expressed. In fact, this appears to be the case for at least one gene directly targeted by miR-9, namely PMP22. PMP22 is predominantly produced in myelinating Schwann cells of the peripheral nervous system (PNS), yet PMP22 mRNA expression is observed in OPCs [Baumann and Pham-Dinh, 2001; Cahoy et al., 2008; Dugas et al., 2006]. Lau et al. [2008] demonstrated that miR-9 directly inhibits the production of PMP22 protein in OL lineage cells. In contrast, miR-9 is not detected in PMP22-expressing Schwann cells [Verrier et al., 2009]. These data illustrate an additional role of miRNAs, serving as 'guardians of the transcriptome' by preventing inappropriately expressed mRNAs from being translated into functional proteins that could detrimentally affect the health of the cell.

Interestingly, additional potential guardians of the transcriptome include the differentiation-promoting miRNAs miR-219 and miR-338. Zhao et al. [2010] point out that these miRNAs are predicted to target the neurogenic transcription factors NeuroD1, Isl1, and Otx2. This raises the possibility that miR-219 and miR-338 may contribute to the tight repression of these genes, which are never expressed in OLs. The fact that miR-219 and miR338 could be involved both in extinguishing OPC gene expression and blocking inappropriate neurogenic gene expression illustrates that miRNAs, by virtue of the wide variety of genes they are capable of targeting, can simultaneously influence distinct aspects of a cell's gene expression program.

\section{miRNA Suppression of Genes Transiently Required during Differentiation}

In addition to its role in promoting OL differentiation and a putative role in preventing inappropriate neurogenic gene expression, miR-219 also contributes to the regulation of gene expression in fully mature OLs. Robust OL expression of miR-219 has consistently been detected in young adult mice (postnatal day P50-60), and the expres-

MicroRNAs in Oligodendrocyte and Schwann Cell Differentiation sion of miR-219 is lost when Dicerl function is specifically ablated in mature OLs in mice containing a tamoxifen-inducible PLP-CreER ${ }^{T}$ gene [Dugas et al., 2010; Shin et al., 2009]. Interestingly, disruption of OL-expressed Dicerl at P14-18 leads to a strong reduction in miR-219 levels in mature OLs by P30, but at this age mutant mice look normal, and only begin to show functional deficits by P60-90, with reduced CNS myelin observed at P180. Why the delay, and what is the reason for the eventual loss of healthy CNS myelin? In these mice, Dicerl function is only disrupted in mature OLs, so OL specification and differentiation should not be adversely affected. Instead, these results indicate a role for mature miRNAs in maintaining healthy myelin, and that miRNAs in mature OLs are more acutely required after myelin has been fully formed by P45-60 [Baumann and Pham-Dinh, 2001], as opposed to during the initial generation of myelin sheaths. This dichotomy may be explained by the fact that a prominent target of miR-219 is ELOVL7 [Shin et al., 2009]. ELOVL7 is an enzyme expressed at high levels in OLs that is involved in the production of very-long-chain fatty acids (VLCFA) [Cahoy et al., 2008; Tamura et al., 2009]. VLCFA is incorporated into PLP as an integral component of the fatty myelin sheath, but overproduction of VLCFA can lead to demyelinating diseases such as Xlinked adrenoleukodystrophy [Dubois-Dalcq et al., 1999]. Potentially, high levels of ELOVL7 activity could be required during active myelin sheath formation, when large amounts of lipid-rich membranes are being produced, but this production would need to be tempered once full axonal myelination is completed. miR-219 may serve this role in mature OLs by reducing the amount of functional ELOVL7 produced from the highly transcribed ELOVL7 locus. In general, these data indicate that another role of miRNAs in OLs, and likely in other cell types, may be to moderate the expression of proteins that are required at high levels for the transformation from immature to fully differentiated phenotype, but whose continued high expression in stable, mature cells may be detrimental. As a consequence of the continued expression of transcription factors that specify the mature state of the cell, expression of these genes would persist, but could be regulated by miRNA suppression of targeted genes.

\section{Loss of Dicer1 Impairs Peripheral Nerve Myelination}

Broad verification for the role of miRNAs in peripheral nerve myelination was recently shown by 5 independent studies of Dicerl-deficient Schwann cells 
Table 1. miRNAs whose expression changed the most in the referenced studies

\begin{tabular}{lll}
\hline Bremer et al. [2010] & Yun et al. [2010] & Verrier et al. [2010] \\
\hline miR-138 & miR-138 & miR-129 \\
miR-140 & miR-338 & miR-145 \\
miR-146b & & miR-193 \\
miR-195 & & miR-222 \\
miR-204 & miR-29a \\
miR-27b & \\
miR-30a & & \\
miR-338-3p & & \\
miR-34a & & \\
\hline
\end{tabular}

[Bremer et al., 2010; Dugas et al., 2010; Pereira et al., 2010; Verrier et al., 2010; Yun et al., 2010]. In 4 in vivo studies in mice, Dicerl was silenced in Schwann cells and peripheral nerve morphology and gene expression profiles were characterized between the ages E12 and P35 [Bremer et al., 2010; Dugas et al., 2010; Pereira et al., 2010; Yun et al., 2010]. While the specific molecular approaches for gene silencing and the methods of analyses used varied among the studies, in each case the ablation of Dicerl within Schwann cells led to glial overproliferation and aberrant myelination. Molecules associated with Schwann cell differentiation were underexpressed, while genes linked to cellular proliferation remained high. Pereira et al. [2010] also detected a severe neurologic phenotype by 5 weeks of age and pronounced axonal degeneration by P24. Similarly, the report by Yun et al. [2010] describes a neuromuscular phenotype consistent with congenital hypomyelination. Depletion of Dicerl beginning at E13 also led to severe myelin defects and severe hind limb paresis by P25 [Bremer et al., 2010; Dugas et al., 2010].

Using an in vitro coculture assay of rat Schwann cells and dorsal root ganglion neurons, Verrier et al. [2010] deleted Dicerl using a Dicerl shRNA lentivirus. In agreement with the aforementioned studies in transgenic mice, inhibition of Dicerl expression reduced the steady-state levels of pro-myelination transcription factors such as Oct6 and Egr2/Krox20, and the myelin protein MPZ. On the other hand, a reduction in Dicerl resulted in increased expression of anti-myelinating proteins, such as c-Jun and Sox 2, an effect that was also reported by Pereira et al. [2010]. In addition, similar to the results in the murine models, Schwann cells with reduced Dicerl expression failed to myelinate axons even after 2 weeks in my- elination-promoting culture conditions [Verrier et al., 2010]. Together, these 5 distinct studies indicate that miRNA biogenesis is critical for Schwann cell myelination and peripheral nerve development. While overall there is good agreement among the studies with regard to myelin defects in the absence of Dicerl, changes in the expression of specific miRNAs are less consistent. Table 1 lists some of the miRNAs that were found to change most significantly in each given paradigm. The discrepancy among the studies is likely due to the specific experimental designs and the developmental stages of analyses. While Dicerl was ablated in a Schwann cell-specific manner in each of the transgenic mouse studies, the mouse miRNA arrays employed whole nerve RNAs [Bremer et al., 2010; Yun et al., 2010]. Therefore, contribution from changes in axonal, endothelial, fibroblast and possibly macrophage-derived miRNAs cannot be ruled out. In addition, the experimental methodologies used to evaluate the levels of miRNAs also differed across the 3 studies (shown in table 1), and included commercial miRNA microarrays, miRNA Northern blots, as well as RT-PCR.

\section{Individual miRNAs in Schwann Cell Biology}

Similar to OLs, the differentiation of Schwann cells and myelination of peripheral nerves involve multifaceted communication between axons and myelinating glia. Distinct from OLs, Schwann cells must establish a one-to-one relationship with specific axonal segments, and also exit the cell cycle prior to the increase in the expression of myelin proteins, including PMP22, MPZ and MBP [Baumann and Pham-Dinh, 2001]. This orchestrated switch in phenotype from a proliferating to a differentiated cell type involves transcriptional and translational gene regulatory mechanisms, including gene repression via miRNAs. As discussed above, one of the first suggested functions of miRNAs in Schwann cell biology came from the discovery that the abundantly expressed oligodendroglial miR-9 has the ability to repress PMP22 [Lau et al., 2008]. PMP22, one of the dosage-sensitive genes in glial cells, is a likely candidate for regulation by miRNAs as its product is a protein whose precise levels are critical for normal Schwann cell function. Indeed, both over- and underexpression of PMP22 have been associated with hereditary demyelinating neuropathies in humans [Chance, 2004].

The discovery that a PMP22-targeting miRNA, namely miR-9, suppresses PMP22 expression in OLs [Lau et al., 
2008] led to the investigation of additional miRNAs with potential regulatory roles in other cell types. Using bioinformatics and microarrays, Verrier et al. [2009] identified miR-29a, miR-381 and miR-140* that are predicted to target the $3^{\prime} \mathrm{UTR}$ of PMP22 and are differentially expressed in actively proliferating as compared to nonproliferating Schwann cells. miR-9 was not detected in these microarrays, nor was it found to be expressed in cultured Schwann cells by RT-PCR or Northern blot. Notably, the levels of miR-29a are downregulated nearly 7 -fold when the cells are promoted to differentiate and express elevated levels of PMP22 [Verrier et al., 2009]. Luciferase reporter assays and real-time RT-PCR experiments confirmed an inverse relationship between miR-29a and PMP22 and demonstrated a reduction in PMP22 mRNA levels when miR-29a is high. On the other hand, inhibition of the endogenous miR-29a increased the steadystate levels of PMP22 mRNA and protein. This inverse functional relationship suggests that miR-29a is an important regulator for PMP22 production in cultured Schwann cells. A role for miR-29a in regulating PMP22 is supported by quantitative RT-PCR studies of whole RNAs isolated from developing rat nerves and from post-crush injury adult mouse nerves [Verrier et al., 2009]. In each of these paradigms, the inverse correlation between miR29a and PMP22 expression supports a functional link between the differentiation state of Schwann cells and their miRNA expression profile, with miR-29a preventing the premature expression of PMP22 in immature Schwann cells. Interestingly, miR-29a is also expressed in OPCs and repressed during OL differentiation [Dugas et al., 2010]. As another predicted target of miR-29a is the CNS myelin protein PLP1, it is tempting to speculate that this miRNA may be playing a parallel role in both the PNS and CNS to block inappropriate production of mature myelin proteins in immature precursors.

Another parallel between myelinating cells of the PNS and CNS is the induction of miR-138 and miR-338 expression in differentiating OLs and Schwann cells [Bremer et al., 2010; Dugas et al., 2010; Yun et al., 2010; Zhao et al., 2010]. Indeed, Yun et al. [2010] found that miR-138 could reduce the expression of Ccnd1, Jun, and Sox2, all genes expressed in immature Schwann cells that are normally repressed during differentiation. These data indicate that the induction of miRNAs upon the initiation of differentiation to rapidly repress immature, proliferation-promoting genes may be a common theme between OLs and Schwann cells, to the extent that a subset of identical miRNAs (miR-138 and miR-338) may play similar roles in both cell types.

MicroRNAs in Oligodendrocyte and Schwann Cell Differentiation

\section{miRNAs in Glial Tumors and Autoimmune Neuropathies}

miRNAs are viewed as master regulators of oncogenes and tumor suppressor genes in a number of cancers [Garofalo and Croce, 2010]. The role of miRNAs in promoting OL differentiation by inhibiting OPC-expressed proliferation-promoting genes may also indicate that miRNA misregulation could contribute to glioma proliferation. Specifically, if OL-expressed miRNAs inhibit cellular proliferation, then loss of these miRNAs could create a permissive environment for tumorigensis. In fact, in analyzed medulloblastoma samples OL-expressed miR-219, miR-138, and miR-192 are all downregulated relative to normal control tissue expression [Ferretti et al., 2009]. An additional link between miRNAs and glial tumors is the recent finding of reduced miR-29 expression in devil facial tumor disease, a transmissible cancer of Schwann cell origin [Murchison et al., 2010]. Cumulatively, these data indicate that reintroduction of Schwann cell or OL-enriched miRNAs into active neural tissue tumors, especially those expressing characteristics of the OPC-OL or Schwann cell lineage, could prove efficacious in blocking tumor progression and/or driving tumor regression.

To gain insights into the role of miRNAs in neural differentiation, the expression and the localization of Dicerl and Argonaute proteins have been examined in neuroblastoma cells that can be differentiated into Schwann or neuron-type cells [Potenza et al., 2009]. In terminally differentiated Schwann cells, the expression of Dicerl and Argonaute proteins was lower as compared to neurons, suggesting that miRNA function is more critical in maintenance of the differentiated neuronal phenotype as compared to Schwann cells. Studies in cultured primary rat Schwann cells agree with this observation, as GW bodies and Dicerl are more prevalent in proliferating as compared to nonproliferating cultures [Verrier et al., 2009].

In conclusion, identification of important miRNA networks in neural tissue development and functioning is rapidly advancing [Hutchison et al., 2009]. A critical role for miRNAs in the human nervous system is supported by clinical reports on patients with autoantibodies to GW182 or to GW/P bodies [Bhanji et al., 2007; Eystathioy et al., 2003]. The most frequent complaint in these patients was motor and sensory neuropathy and ataxia. These data likely indicate that miRNAs are not only critical during neural development, but that they are also important in maintaining a healthy nervous system. The widespread impact of miRNA expression in the mamma-

Dev Neurosci 2011;33:14-20 
lian nervous system, including the myelinating cells of the CNS and PNS, indicates that this recently identified class of molecules may offer novel avenues for future therapeutic developments in a wide variety of dysmyelinating and other neurodegenerative diseases.

\section{Acknowledgements}

L.N. is supported by the National Institutes of Health NS041012 and the Trigeminal Neuralgia Association Facial Pain Research Program; J.C.D. is supported by the Myelin Repair Foundation and the National Multiple Sclerosis Society RG4059A8.

\section{References}

Bartel DP (2004): MicroRNAs: genomics, biogenesis, mechanism, and function. Cell 116: 281-297.

Bartel DP (2009): MicroRNAs: target recognition and regulatory functions. Cell 136:215-233.

-Baumann N, Pham-Dinh D (2001): Biology of oligodendrocyte and myelin in the mammalian central nervous system. Physiol Rev 81: 871-927.

-Bernstein E, Kim SY, Carmell MA, Murchison EP, Alcorn H, Li MZ, Mills AA, Elledge SJ, Anderson KV, Hannon GJ (2003): Dicer is essential for mouse development. Nat Genet 35:215-217.

Bhanji RA, Eystathioy T, Chan EK, Bloch DB, Fritzler MJ (2007): Clinical and serological features of patients with autoantibodies to GW/P bodies. Clin Immunol 125:247-256.

-Bremer J, O'Connor T, Tiberi C, Rehrauer H, Weis J, Aguzzi A (2010): Ablation of Dicer from murine Schwann cells increases their proliferation while blocking myelination. PLoS One 5:e12450.

Budde H, Schmitt S, Fitzner D, Opitz L, SalinasRiester G, Simons M (2010): Control of oligodendroglial cell number by the miR-17-92 cluster. Development 137:2127-2132.

Cahoy JD, Emery B, Kaushal A, Foo LC, Zamanian JL, Christopherson KS, Xing Y, Lubischer JL, Krieg PA, Krupenko SA, Thompson WJ, Barres BA (2008): A transcriptome database for astrocytes, neurons, and oligodendrocytes: a new resource for understanding brain development and function. J Neurosci 28:264-278.

Chance PF (2004): Genetic evaluation of inherited motor/sensory neuropathy. Suppl Clin Neurophysiol 57:228-242.

Dubois-Dalcq M, Feigenbaum V, Aubourg P (1999): The neurobiology of X-linked adrenoleukodystrophy, a demyelinating peroxisomal disorder. Trends Neurosci 22:4-12.

Dugas JC, Cuellar TL, Scholze A, Ason B, Ibrahim A, Emery B, Zamanian JL, Foo LC, McManus MT, Barres BA (2010): Dicerl and miR-219 are required for normal oligodendrocyte differentiation and myelination. Neuron 65:597-611.

Dugas JC, Tai Y-C, Speed TP, Ngai J, Barres BA (2006): Functional genomic analysis of oligodendrocyte differentiation. J Neurosci 26: 10967-10983.

-Eystathioy T, Chan EK, Takeuchi K, Mahler M, Luft LM, Zochodne DW, Fritzler MJ (2003): Clinical and serological associations of autoantibodies to GW bodies and a novel cytoplasmic autoantigen GW182. J Mol Med 81: 811-818.
Ferretti E, De Smaele E, Po A, Di Marcotullio L, Tosi E, Espinola MS, Di Rocco C, Riccardi R, Giangaspero F, Farcomeni A, Nofroni I, Laneve P, Gioia U, Caffarelli E, Bozzoni I, Screpanti I, Gulino A (2009): MicroRNA profiling in human medulloblastoma. Int J Cancer 124:568-577.

Garofalo M, Croce CM (2011): microRNAs: master regulators as potential therapeutics in cancer. Annu Rev Pharmacol Toxicol 51:25-43.

Hutchison ER, Okun E, Mattson MP (2009): The therapeutic potential of microRNAs in nervous system damage, degeneration, and repair. Neuromolecular Med 11:153-161.

Kawase-Koga Y, Otaegi G, Sun T (2009): Different timings of Dicer deletion affect neurogenesis and gliogenesis in the developing mouse central nervous system. Dev Dyn 238: 2800-2812.

Lau P, Verrier JD, Nielsen JA, Johnson KR, Notterpek L, Hudson LD (2008): Identification of dynamically regulated microRNA and mRNA networks in developing oligodendrocytes. J Neurosci 28:11720-11730.

Lin ST, Fu YH (2009): miR-23 regulation of la$\mathrm{min} \mathrm{B} 1$ is crucial for oligodendrocyte development and myelination. Dis Model Mech 2: $178-188$.

Murchison EP, Tovar C, Hsu A, Bender HS, Kheradpour P, Rebbeck CA, Obendorf D, Conlan C, Bahlo M, Blizzard CA, Pyecroft S, Kreiss A, Kellis M, Stark A, Harkins TT, Marshall Graves JA, Woods GM, Hannon GJ, Papenfuss AT (2010): The Tasmanian devil transcriptome reveals Schwann cell origins of a clonally transmissible cancer. Science 327:84-87.

Padiath QS, Saigoh K, Schiffmann R, Asahara H, Yamada T, Koeppen A, Hogan K, Ptacek LJ, Fu YH (2006): Lamin B1 duplications cause autosomal dominant leukodystrophy. Nat Genet 38:1114-1123.

Paintlia AS, Paintlia MK, Singh AK, Orak JK, Singh I (2010): Activation of PPAR-gamma and PTEN cascade participates in lovastatinmediated accelerated differentiation of oligodendrocyte progenitor cells. Glia 58:16691685.

Pereira JA, Baumann R, Norrmen C, Somandin C, Miehe M, Jacob C, Luhmann T, HallBozic H, Mantei N, Meijer D, Suter U (2010): Dicer in Schwann cells is required for myelination and axonal integrity. J Neurosci 30: 6763-6775.

Potenza N, Papa U, Russo A (2009): Differential expression of Dicer and Argonaute genes during the differentiation of human neuroblastoma cells. Cell Biol Int 33:734-738.
Reinhart BJ, Slack FJ, Basson M, Pasquinelli AE, Bettinger JC, Rougvie AE, Horvitz HR, Ruvkun G (2000): The 21-nucleotide let-7 RNA regulates developmental timing in Caenorhabditis elegans. Nature 403:901-906.

Shin D, Shin JY, McManus MT, Ptacek LJ, Fu YH (2009): Dicer ablation in oligodendrocytes provokes neuronal impairment in mice. Ann Neurol 66:843-857.

Stolt CC, Schlierf A, Lommes P, Hillgartner S, Werner T, Kosian T, Sock E, Kessaris N, Richardson WD, Lefebvre V, Wegner M (2006): SoxD proteins influence multiple stages of oligodendrocyte development and modulate SoxE protein function. Dev Cell 11:697-709.

-Tamura K, Makino A, Hullin-Matsuda F, Kobayashi T, Furihata M, Chung S, Ashida S, Miki T, Fujioka T, Shuin T, Nakamura Y, Nakagawa H (2009): Novel lipogenic enzyme ELOVL7 is involved in prostate cancer growth through saturated long-chain fatty acid metabolism. Cancer Res 69:8133-8140.

- Verrier JD, Lau P, Hudson L, Murashov AK, Renne R, Notterpek L (2009): Peripheral myelin protein 22 is regulated post-transcriptionally by miRNA-29a. Glia 57:1265-1279.

-Verrier JD, Semple-Rowland S, Madorsky I, Papin JE, Notterpek L (2010): Reduction of Dicer impairs Schwann cell differentiation and myelination. J Neurosci Res 88:25582568.

Watkins TA, Emery B, Mulinyawe S, Barres BA (2008): Distinct stages of myelination regulated by gamma-secretase and astrocytes in a rapidly myelinating CNS coculture system. Neuron 60:555-569.

Wienholds E, Kloosterman WP, Miska E, Alvarez-Saavedra E, Berezikov E, de Bruijn E, Horvitz HR, Kauppinen S, Plasterk RH (2005): MicroRNA expression in zebrafish embryonic development. Science 309:310311.

Wu L, Belasco JG (2008): Let me count the ways: mechanisms of gene regulation by miRNAs and siRNAs. Mol Cell 29:1-7.

-Yun B, Anderegg A, Menichella D, Wrabetz L, Feltri ML, Awatramani R (2010): MicroRNA-deficient Schwann cells display congenital hypomyelination. J Neurosci 30:77227728.

Zhao X, He X, Han X, Yu Y, Ye F, Chen Y, Hoang T, Xu X, Mi QS, Xin M, Wang F, Appel B, Lu QR (2010): MicroRNA-mediated control of oligodendrocyte differentiation. Neuron 65: 612-626. 